\title{
Quantum Renormalization of the Spin Hall Effect
}

\author{
Bo $\mathrm{Gu}^{1,2}$, Jing-Yu Gan ${ }^{3}$, Nejat Bulut ${ }^{4}$, Timothy Ziman ${ }^{5}$, \\ Guang-Yu Guo ${ }^{6,7}$, Naoto Nagaosa ${ }^{8,9}$, and Sadamichi Maekawa ${ }^{1,2}$ \\ ${ }^{1}$ Advanced Science Research Center, Japan Atomic Energy Agency, Tokai 319-1195, Japan \\ ${ }^{2}$ JST, CREST, 3-Sanbancho,Chiyoda-ku, Tokyo 102-0075, Japan \\ ${ }^{3}$ Institute of Physics, Chinese Academy of Sciences, P.O. Box 603, Beijing 100190, China \\ ${ }^{4}$ Department of Physics, Izmir Institute of Technology, Urla 35430, Turkey \\ ${ }^{5}$ CNRS and Institut Laue Langevin, Boîte Postale 156, F-38042 Grenoble Cedex 9, France \\ ${ }^{6}$ Graduate Institute of Applied Physics, National Chengchi University, Taipei 116, Taiwan \\ ${ }^{7}$ Department of Physics, National Taiwan University, Taipei 106, Taiwan \\ ${ }^{8}$ Department of Applied Physics, The University of Tokyo, Tokyo 113-8656, Japan \\ ${ }^{9}$ Cross-Correlated Materials Research Group and Correlated Electron Research Group, RIKEN-ASI, Wako 315-0198, Japan
}

\begin{abstract}
By quantum Monte Carlo simulation of a realistic multiorbital Anderson impurity model, we study the spin-orbit interaction (SOI) of an Fe impurity in Au host metal. We show, for the first time, that the SOI is strongly renormalized by the quantum spin fluctuation. Based on this mechanism, we can explain why the gigantic spin Hall effect in Au with Fe impurities was observed in recent experiment, while it is not visible in the anomalous Hall effect. In addition, we show that the SOI is strongly renormalized by the Coulomb correlation U. Based on this picture, we can explain past discrepancies in the calculated orbital angular momenta for an Fe impurity in an Au host.
\end{abstract}

PACS numbers: $71.70 . \mathrm{Ej}, 75.30 . \mathrm{Kz}, 75.40 . \mathrm{Mg}$

When a magnetic impurity with $d$ orbitals is put into a normal metal with $s$ - or $p$-conduction bands, it forms a virtual bound state hybridized with the conduction states, leading to a broadening with width $\Delta$ (hybridization energy) which is typically of the order of eV. This energy scale competes with the Coulomb energy $U$ between the electrons in the $d$ orbitals, which is also of the order of eV. The latter tends to produce the spin moment, while the former induces quantum fluctuation of that spin moment, leading to the spin singlet. The competition between these two interactions defines the time scale, or equivalently the energy scale, of the quantum spin fluctuation, i.e., the Kondo temperature $T_{K}$, which can be much lower than both $\Delta$ and $U$ [1]. In the language of the renormalization group, this is described by the energy dependent scaling of the various operators. This means that even a weak interaction can be amplified in the low energy or temperature scale and compete with the much larger energy scale due to the correlation $U$.

In real systems, the $d$ orbitals have fivefold degeneracy and these orbital degrees of freedom have often been neglected in the analysis of the experimental results. The reasoning is that either the crystal field splitting $\Delta \varepsilon$ is much smaller than the hybridization energy $\Delta$, or that it is in the limit with $\Delta \varepsilon$ much larger than the Kondo temperature $T_{K}$. However, the naive comparison between the bare interaction strengths is dangerous since these are scale-dependent running coupling constants. Similar nontrivial behavior can also be expected for the relativistic spin-orbit interaction.

Orbital degrees of freedom in the impurity scattering lead to intriguing phenomena such as the anomalous Hall effect (AHE) and the spin Hall effect (SHE). A charge current perpendicular to the applied electric field is produced in ferromagnetic metals (AHE), while a spin current rather than a charge current, is induced in semiconductors and metals without magnetism (SHE). An extrinsic mechanism of these two effects arises from the skew scattering, i.e., spin-dependent deflection of the scattered electrons due to the spin-orbit interaction. It has been shown in the case of the AHE that the resonant skew scattering due to the virtual bound $d$ states leads to a large Hall angle, of the order of 0.01 [2], compared with the typical value of $10^{-3}$. In the AHE, the spin fluctuation is quenched, owing to the ferromagnetism or the external magnetic field, and the ratio of the spin-orbit interaction $\lambda$ and $\Delta$ basically determines the Hall angle. On the other hand, in the SHE, the spin fluctuation is active and the Kondo physics can be relevant to the resonant skew scattering. Therefore, a crucial question is whether the Kondo effect and quantum spin fluctuation can produce an even larger spin Hall angle compared with the AHE.

An important clue to this question comes from an experiment by Seki et al. [3] on $\mathrm{Au} / \mathrm{FePt}$, in which a spin Hall angle of 0.114 was observed. Motivated by this experiment, some of the present authors studied the Fe impurity in Au by a first-principles calculation [4]. The Kondo effect of Fe impurities is a historic problem, with a low $T_{K}$ around $0.4 \mathrm{~K}$ [5], high electric resistance at room temperature [1], and an AHE with Hall angle of the order of 0.01 [2]. However, a simple fivefold degeneracy of the orbitals has been assumed to analyze the experiments. In Ref. [4], on the other hand, the orbital-dependent Kondo effect of $\mathrm{Fe}$ in $\mathrm{Au}$ was proposed to explain the nature of the experimentally observed giant spin Hall signals [4]: It was argued that the $e_{g}$ orbitals of Fe are in the Kondo limit and $t_{2 g}$ orbitals are in the mixed-valence region. The enhancement of the spin-orbit interaction by electron 
correlation in the $t_{2 g}$ orbitals leads to the giant spin Hall effect. However this proposal has been challenged theoretically by Ref.[6], which suggests an effective 3-channel Kondo model, involving local and band electrons of $t_{2 g}$ symmetry, and also experimentally by the x-ray MCD [7], which obtained a rather small value of the orbital angular momentum in contrast to the large value calculated in Ref. [4].

In order to resolve this confusing situation and quantify the mechanism for the enhanced spin Hall effect, it is essential to treat the quantum fluctuations of the spins and orbitals systematically. This is impossible in the first-principles calculation, which assumes ordered spin and orbital moments. In this Letter, we overcome this difficulty by using the Hirsch-Fye quantum Monte Carlo (QMC) simulation 8], combined with the density functional theory (DFT) [9, 10], to study the renormalization due to correlation effects. First, a single-impurity multiorbital Anderson model [11] is formulated within the DFT for determining the host band structure, the impurity levels, and the impurity-host hybridization. Second, the magnetic behaviors of the Anderson impurity at finite temperatures are calculated by QMC.

The single-impurity multiorbital Anderson model is defined as

$$
\begin{aligned}
H & =\sum_{\mathbf{k}, \alpha, \sigma} \epsilon_{\alpha}(\mathbf{k}) c_{\mathbf{k} \alpha \sigma}^{\dagger} c_{\mathbf{k} \alpha \sigma}+\sum_{\mathbf{k}, \alpha, \xi, \sigma}\left(V_{\xi \mathbf{k} \alpha} d_{\xi \sigma}^{\dagger} c_{\mathbf{k} \alpha \sigma}+H . c .\right) \\
& +\sum_{\xi, \sigma} \epsilon_{\xi} n_{\xi \sigma}+U \sum_{\xi} n_{\xi \uparrow} n_{\xi \downarrow} \\
& +\frac{U^{\prime}}{2} \sum_{\xi \neq \xi^{\prime}, \sigma, \sigma^{\prime}} n_{\xi \sigma} n_{\xi^{\prime} \sigma^{\prime}}-\frac{J}{2} \sum_{\xi \neq \xi^{\prime}, \sigma} n_{\xi \sigma} n_{\xi^{\prime} \sigma}
\end{aligned}
$$

where $c_{\mathbf{k} \alpha \sigma}^{\dagger}\left(c_{\mathbf{k} \alpha \sigma}\right)$ is the creation (annihilation) operator of the conduction electron with wavevector $\mathbf{k}$ and spin $\sigma$ in the band $\alpha, d_{\xi \sigma}^{\dagger}\left(d_{\xi \sigma}\right)$ is the creation (annihilation) operator of the localized electron at the impurity site with orbital $\xi$ and spin $\sigma$, and $n_{\xi \sigma}=d_{\xi \sigma}^{\dagger} d_{\xi \sigma}$. The host energy band $\epsilon_{\alpha}(\mathbf{k})$, the impurity energy levels $\epsilon_{\xi}$, and the impurity-host hybridization $V_{\xi \mathbf{k} \alpha}$, as the one-body problems, can be properly obtained by the DFT calculations. $U\left(U^{\prime}\right)$ is the on-site Coulomb repulsion within (between) the orbitals of the impurity, and $J$ is the Hund coupling between the orbitals of the impurity. These many-body interactions can be exactly treated by the QMC calculations, and thus it becomes possible to accurately study the quantum fluctuations of the spins and orbitals of the impurity. Considering the parameters used in the previous calculations for $\mathrm{Fe}$ in $\mathrm{Au}$ [4], and the relationship $U=U^{\prime}+2 J[12$, in our following QMC calculations, we use the values of $U=5 \mathrm{eV}, J=0.9 \mathrm{eV}$, and $U^{\prime}=$ $3.2 \mathrm{eV}$ for most cases, but we shall vary the values for a few cases in order to clarify the role of correlations and reconcile with past calculations.

Our DFT calculations are done by the code QUANTUMESPRESSO [13]. To calculate the impurity-host hybridization, we consider the supercell $\mathrm{Au}_{26} \mathrm{Fe}$, where the
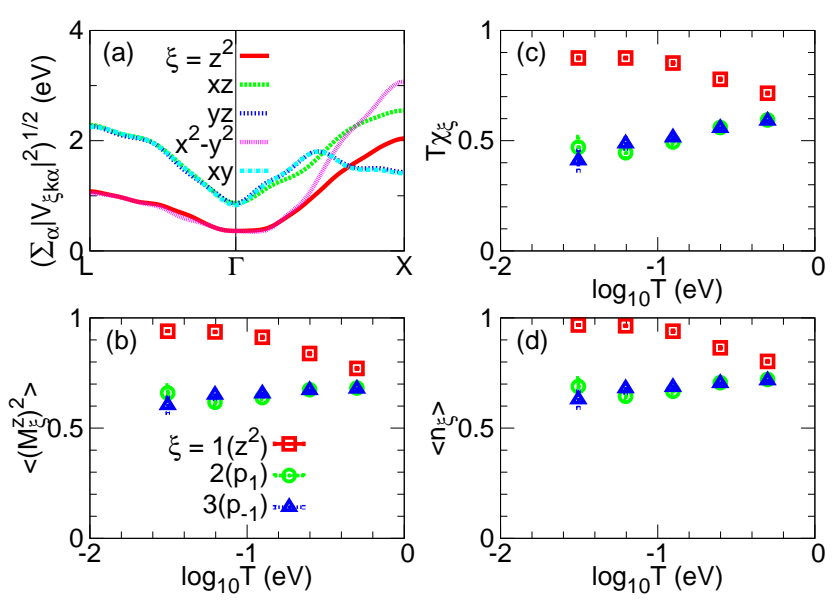

FIG. 1. (color online) (a) Hybridization between the $\xi$ orbitals of an Fe impurity and an Au host. We show the LDA result including five $d$ orbitals. (b) Temperature dependence of the square of the magnetic moment $\left\langle\left(M_{\xi}^{z}\right)^{2}\right\rangle$, (c) the temperature times susceptibility $T \chi_{\xi}$, and (d) the occupation number $\left\langle n_{\xi}\right\rangle$ of the $\xi$ orbitals of the Fe impurity. (b)-(d) are the QMC results including three $d$ orbitals. See text for details.

exchange-correlation interactions are described by the Perdew-Zunger local density approximation (LDA), and the electron-ion interactions are represented by the RabeRappe-Kaxiras-Joannopoulos ultrasoft pseudopotentials. $4 \times 4 \times 4$ Monshorst-Pack $\mathbf{k}$ points are used. Kinetic energy cutoff for wavefunctions and charge density are taken as 30 and $300 \mathrm{Ry}$, respectively.

Figure 1(a) shows the hybridization between $\xi$ orbitals of $\mathrm{Fe}$ impurity and $\mathrm{Au}$ host, where the hybridization matrix element has the form of $V_{\xi \mathbf{k} \alpha} \equiv\left\langle\varphi_{\xi}\left|H_{0}\right| \Psi_{\alpha}(\mathbf{k})\right\rangle$ $=\frac{1}{\sqrt{N}} \sum_{p, \mathbf{n}} e^{i \mathbf{k} \cdot \mathbf{n}} a_{\alpha p}(\mathbf{k})\left\langle\varphi_{\xi}\left|H_{0}\right| \varphi_{p}(\mathbf{n})\right\rangle . \quad H_{0}$ is the oneparticle part of Eq. (11), $\varphi_{\xi}$ is the $\xi$ state of Fe impurity, and $\Psi_{\alpha}(\mathbf{k})$ is the Au host state with wavevector $\mathbf{k}$ and band index $\alpha$, which is expanded by atomic orbitals $\varphi_{p}(\mathbf{n})$ with orbital index $p$ and site index $\mathbf{n}, a_{\alpha p}(\mathbf{k})$ are expansion coefficients, $\left\langle\varphi_{\xi}\left|H_{0}\right| \varphi_{p}(\mathbf{n})\right\rangle$ are mixing integrals, and $N$ is the number of lattice sites. It is observed that, at the $\Gamma$ point $(\mathbf{k}=0)$, the hybridization value of the $\xi=e_{g}\left(z^{2}, x^{2}-y^{2}\right)$ orbitals of the Fe impurity is smaller than that of the $\xi=t_{2 g}(x z, y z, x y)$ orbitals.

Based on the above DFT/LDA calculation, we can determine approximate impurity levels $\epsilon_{\xi}=-1.9 \mathrm{eV}$ for $\xi$ $=e_{g}\left(z^{2}, x^{2}-y^{2}\right)$ and $\epsilon_{\xi}=-1.8 \mathrm{eV}$ for $\xi=t_{2 g}(x z, y z, x y)$ with zero Fermi energy, where the Coulomb terms included in the LDA calculations have been subtracted as in Refs. [14, 15]. The crystal field splitting $\Delta \varepsilon=0.1 \mathrm{eV}$ is in agreement with the previous LDA calculations [4, 6].

To study the renormalization due to the correlation effect for (i) the crystal field splitting and (ii) the relativistic spin-orbit interaction, the magnetic behaviors of Eq. (11) are calculated by the Hirsch-Fye QMC simulation for the infinite host system [8]. Owing to calculation constraints, we simplify it to a three-orbital model 
with one $e_{g}$ and two $t_{2 g}$ orbitals, which captures the essential physics. The $e_{g}$ orbital is arbitrarily chosen as $z^{2}$, and the two $t_{2 g}$ orbitals are chosen as $p_{1}$ and $p_{-1}$, where the notation corresponds to the transformational properties of $t_{2 g}$ orbitals equivalent to effective $p$ orbitals [16]: $p_{1} \equiv-\frac{1}{\sqrt{2}}(x z-i y z), p_{0} \equiv-i x y$, and $p_{-1} \equiv-\frac{1}{\sqrt{2}}(x z+i y z)$. The following results are obtained with more than $10^{5}$ Monte Carlo sweeps and the Matsubara time step $\Delta \tau=0.25$.

Figures 1(b)-(d) show the temperature dependence of the square of the magnetic moment $\left\langle\left(M_{\xi}^{z}\right)^{2}\right\rangle$, the temperature times susceptibility $T \chi_{\xi}$, and the occupation number $\left\langle n_{\xi}\right\rangle$, which are defined as $M_{\xi}^{z}=n_{\xi \uparrow}-n_{\xi \downarrow}, \chi_{\xi}$ $=\int_{0}^{\beta} d \tau\left\langle M_{\xi}^{z}(\tau) M_{\xi}^{z}(0)\right\rangle, n_{\xi}=n_{\xi \uparrow}+n_{\xi \downarrow}$, respectively, and the impurity levels $\epsilon_{\xi}=-1.9\left(\xi=z^{2}\right)$ and $-1.8 \mathrm{eV}$ $\left(\xi=p_{1}, p_{-1}\right)$ are used. It is found that the $e_{g}\left(t_{2 g}\right)$ orbital has a larger (smaller) magnetic moment, a much larger (smaller) susceptibility with a much smaller (larger) Kondo temperature, and a larger (smaller) occupation number. In addition, if we repeat the QMC calculation with the degenerate impurity levels by hand, $\epsilon_{\xi}=$ $-1.85 \mathrm{eV}$ for $\xi=z^{2}, p_{1}$ and $p_{-1}$, nearly the same behaviors are observed. Thus it is clear that the orbital-dependent Kondo effect comes mainly from the renormalization of the impurity-host hybridization by correlations.

Next, we study problem (ii), the renormalization of the relativistic spin-orbit interaction due to the correlation. For simplicity, we consider only the $z$ component,

$$
H_{s o}=(\lambda / 2)\left(\ell^{z} \sigma^{z}\right), \ell^{z} \sigma^{z} \equiv n_{2 \uparrow}-n_{2 \downarrow}-n_{3 \uparrow}+n_{3 \downarrow},(2)
$$

where $\mathbf{l}^{z}$ is the $3 \times 3 z$ component of the angular moment matrix of $\ell=1$, and $\sigma^{z}$ is the $2 \times 2 z$ component of Pauli matrix. $\xi=2(3)$ denotes the $p_{1}\left(p_{-1}\right)$ orbital. We add Eq.(2) to Eq.(11), where the parameters in Eq.(11) are taken as the same values of Figs. 1(b)-(d). For an $\mathrm{Fe}$ atom, the realistic value of the spin-orbit interaction is $\lambda=75 \mathrm{meV}$ [17]. We also show the results with the smaller value of $\lambda=40 \mathrm{meV}$ for comparison. Considering that the value of $\lambda$ is 2 orders of magnitude less than that of impurity energies $\epsilon_{\xi}$, it is not surprising that the temperature dependence of the square of the magnetic moment $\left\langle\left(M_{\xi}^{z}\right)^{2}\right\rangle$, the susceptibility $T \chi_{\xi}$, and the occupation number $\left\langle n_{\xi}\right\rangle$ are nearly the same as those of Figs. 1(b)-(d). In contrast, as displayed in Fig. 2, a nonzero spin-orbit correlation function $\left\langle\ell^{z} \sigma^{z}\right\rangle$ appears when the spin-orbit interaction within the $t_{2 g}$ orbitals of the Fe impurity is included. At temperature $T=360 \mathrm{~K}$, the lower limit of our present calculations, we have $\left\langle\ell^{z} \sigma^{z}\right\rangle \cong-0.44$ with $\lambda=75 \mathrm{meV}$, and $\left\langle\ell^{z} \sigma^{z}\right\rangle \cong-0.3$ with $\lambda=40 \mathrm{meV}$.

To compare the QMC results with the experiment, we calculate the spin Hall angle $\gamma_{s}$ as follows. Since we consider only two $t_{2 g}$ orbitals with the $z$-component of the orbital angular moment $\ell^{z}= \pm 1$, the spin-orbit interaction within the $t_{2 g}$ orbitals gives rise to the difference in the occupation numbers between the parallel $\left(n_{P}\right)$ and antiparallel $\left(n_{A P}\right)$ states of the spin and angular momenta. These occupation numbers are related to the phase shifts

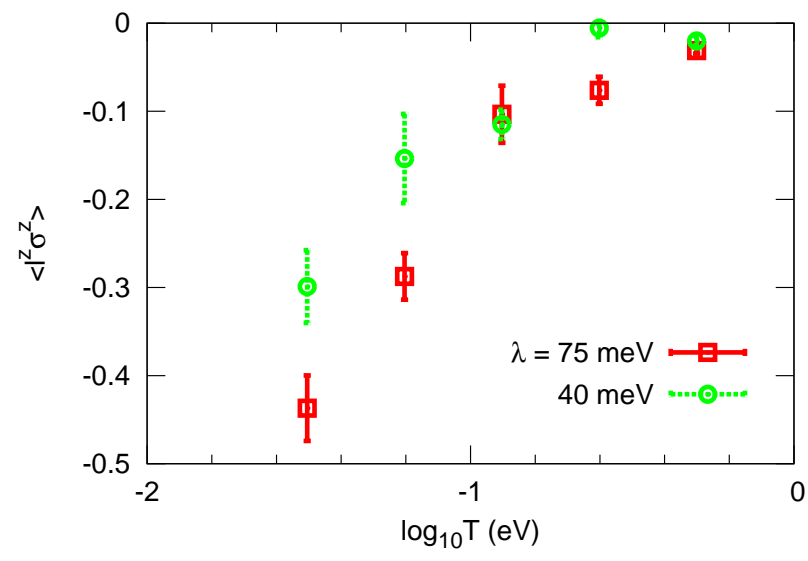

FIG. 2. (color online) Temperature dependence of spin-orbit interactions for an Fe impurity in an Au host. The parameter $\lambda$ in Eq. (2) is taken as a realistic $75 \mathrm{meV}$ [17], and as 40 $\mathrm{meV}$ for comparison.

$\delta_{P}$ and $\delta_{A P}$, respectively as $n_{P(A P)}=\delta_{P(A P)} / \pi$. These quantities can be estimated as

$$
\pi\left\langle\ell^{z} \sigma^{z}\right\rangle=\delta_{P}-\delta_{A P}, \pi\left\langle n_{2}\right\rangle+\pi\left\langle n_{3}\right\rangle=\delta_{P}+\delta_{A P},
$$

which are given in Fig. 2 and $1(\mathrm{~d})$, respectively. Putting $\left\langle\ell^{z} \sigma^{z}\right\rangle=-0.44$ for $\lambda=75 \mathrm{meV}$, and $\left\langle n_{2}\right\rangle=\left\langle n_{3}\right\rangle=0.65$, we obtain $\delta_{P}=1.35$ and $\delta_{A P}=2.73$. Taking into account the estimate $\delta_{1} \cong 0.1$ of the phase shift for p-wave scattering, and applying the equation [4] $\gamma_{s}=6 \operatorname{Im}\left[\left(e^{-2 i \delta_{1}}-\right.\right.$ 1) $\left.\left(e^{2 i \delta_{P}}-e^{2 i \delta_{A P}}\right)\right] /\left[25-15 \cos 2 \delta_{P}-10 \cos 2 \delta_{A P}\right]$, the spin Hall angle is thus obtained as $\gamma_{s} \cong 0.055$, comparable to that observed in recent experiment.

To further understand how correlations renormalize the effective relativistic spin-orbit interaction, we will now vary the correlation energy and the spin polarization, while keeping the temperature $T=360 \mathrm{~K}$ and spinorbit interaction $\lambda=75 \mathrm{meV}$ fixed.

Effect of correlation $U$ : For each $U$, we keep $J / U=$ $0.9 / 5$ and $U=U^{\prime}+2 J$. In Fig. 3 with $\left\langle\left(M_{2}^{z}+M_{3}^{z}\right) / 2\right\rangle=$ 0 , we see that the spin-orbit correlation function $\left|\left\langle\ell^{z} \sigma^{z}\right\rangle\right|$ dramatically decreases as $U$ decreases. This demonstrates clearly how the correlation $U$ renormalizes the relativistic spin-orbit interaction. The results also explain past discrepancies in the calculated orbital angular momenta for an Fe impurity in an Au host: A large value is calculated with $U=5 \mathrm{eV}$ [4], while much smaller ones are obtained with $U=3$ or $2 \mathrm{eV}[\underline{6},[18]$. The virtual bound states of $t_{2 g}$ have majority and minority parts, whose contributions to $\left\langle\ell^{z} \sigma^{z}\right\rangle$ are opposite in sign. Increasing $U$ will push up the minority part away from the Fermi energy, decrease its contribution to $\left\langle\ell^{z} \sigma^{z}\right\rangle$, and hence increase the total $\left\langle\ell^{z} \sigma^{z}\right\rangle$.

Effect of spin polarization: To see clearly the effects of quantum fluctuations, it is instructive to study the effects of polarizing the Fe impurity spin, which can quench quantum fluctuations of the spins and orbitals. We apply a magnetic field $h$ by hand on the orbitals $1\left(z^{2}\right), 2\left(p_{1}\right)$, 


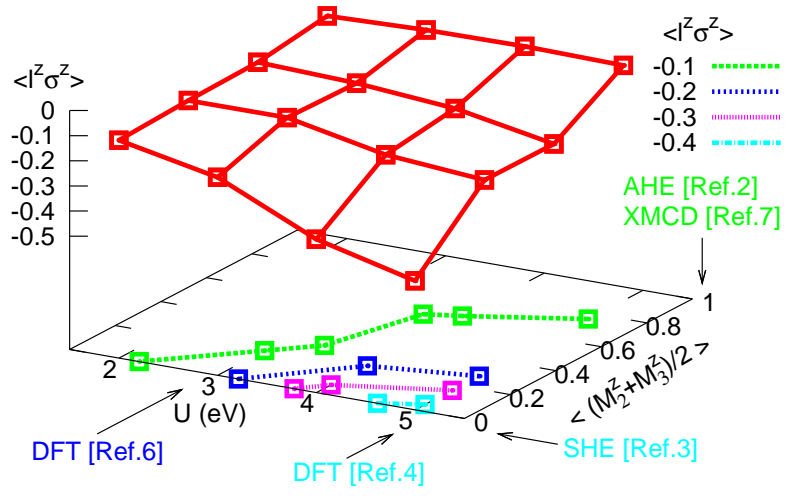

FIG. 3. (color online) For an Fe impurity in an Au host, we show the spin-orbit interaction $\left\langle\ell^{z} \sigma^{z}\right\rangle$ as a function of correlation energy $U$ and spin polarization $\left\langle\left(M_{2}^{z}+M_{3}^{z}\right) / 2\right\rangle$. The QMC results of $\left\langle\ell^{z} \sigma^{z}\right\rangle$ are shown as red squares. In the phase diagram plane of $U$ to $\left\langle\left(M_{2}^{z}+M_{3}^{z}\right) / 2\right\rangle$, the different colors denote the different values of $\left\langle\ell^{z} \sigma^{z}\right\rangle$. Here, $T=360 \mathrm{~K}$ and $\lambda=75 \mathrm{meV}$ are fixed.

and $3\left(p_{-1}\right)$ of the Fe impurity,

$$
H_{f}=-h\left(n_{1 \uparrow}-n_{1 \downarrow}+n_{2 \uparrow}-n_{2 \downarrow}+n_{3 \uparrow}-n_{3 \downarrow}\right),
$$

where $h$ may be of a fraction of $\mathrm{eV}$ in order to polarize the spins at the temperature of $360 \mathrm{~K}$. We add Eq. (4) to the previous Hamiltonian with the parameters in Eq.(1) taken to be the same as in Fig. 2. It is found that, in Fig. 3 with $U=5 \mathrm{eV}$, when $h$ is set up to $0.8 \mathrm{eV}$ to make the spins $\left\langle M_{\xi}^{z}\right\rangle$ of $\xi=1\left(z^{2}\right), 2\left(p_{1}\right)$ and $3\left(p_{-1}\right)$ gradually polarize near the saturated value 1 , the spinorbit correlation function $\left|\left\langle\ell^{z} \sigma^{z}\right\rangle\right|$ gradually decreases to 0 . Thus, the quantum fluctuation of the spin is crucially important in producing an even larger spin Hall angle than the Hall angle of the AHE. What happens is that the quantum fluctuation of the spin leads to the motional narrowing of the spin-orbit coupled virtual bound states, reducing the hybridization energy with the conduction electrons and hence the energy width $\Delta$. So, the Hall angle will increase with decreasing $\Delta[2]$.

Phase diagram: Combining the above two parameters, our QMC results for the spin-orbit correlation function $\left\langle\ell^{z} \sigma^{z}\right\rangle$ are noted as red squares in Fig. 3. In the phase diagram plane of the correlation $U$ to the spin polarization $\left\langle\left(M_{2}^{z}+M_{3}^{z}\right) / 2\right\rangle$, as also noted in Fig. 3, the different colors note the different values of $\left\langle\ell^{z} \sigma^{z}\right\rangle$. It is seen that the areas with large spin-orbit correlation should possess strong correlation energy $U$ and weak spin polarization. The measurement of the orbital angular momentum by x-ray MCD involves the application of a sum rule for atomic wavefunctions: In order to eliminate interference between different impurities, it is necessary to polarize with an external magnetic field at low temperatures [7]. The spin Hall angle measurement [3] was done in a spinunpolarized state and at room temperature. From our phase diagram in Fig. 3, it is suggested that the orbital angular momentum in a spin polarized state could be much smaller than that in a spin-unpolarized state. We emphasize that this phase diagram is obtained for temperatures comparable to room temperature, as is relevant for spin Hall applications; for quantitative comparison of very low temperature measurements, such as x-ray MCD and phase decoherence, the scales can be renormalized.

To conclude, we have studied the SHE for an Fe impurity in Au host by QMC simulation of a multiorbital Anderson impurity model. Our phase diagram with respect to correlation and spin polarization shows that large spinorbit correlations occur for strong correlation $U$ and weak spin polarization, which, in principle, may resolve the current, somewhat confusing, situation of experiments and calculations. More generally, we can, for the first time, quantify an essential difference between the AHE and the SHE. While we have focused on the specific case of $\mathrm{Fe}$ in $\mathrm{Au}$, the results suggest a more general mechanism, and constraints on parameters, for obtaining a large SHE by impurities.

This work was supported by Grant-in-Aids for Scientific Research in the priority area "Spin current" with No. 19048015, No. 19048009, No. 19048008, and No. 21244053, the Next Generation Super Computing Project, the Nanoscience program from MEXT of Japan, the Funding Program for World-Leading Innovative R\&D on Science and Technology (FIRST Program), and NSC of Taiwan. T.Z. is grateful to the ICC-IMR.
[1] G. Gruener et al., Rep. Prog. Phys. 37, 1497 (1974).

[2] A. Fert et al., J. Mag. Mag. Mat. 24, 231 (1981).

[3] T. Seki et. al., Nature Mater. 7, 125 (2008).

[4] G. Y. Guo et al., Phys. Rev. Lett. 102, 036401 (2009).

[5] J. W. Loram et al., Phys. Rev. B 2, 857 (1970).

[6] T. A. Costi et al., Phys. Rev. Lett. 102, 056802 (2009).

[7] W. D. Brewer et al. Phys. Rev. Lett. 93, 077205 (2004).

[8] J. E. Hirsch and, Phys. Rev. Lett. 56, 2521 (1986).

[9] P. Hohenberg and, Phys. Rev. 136, B864 (1964).

[10] W. Kohn and, Phys. Rev. 140, A1133 (1965).

[11] P. W. Anderson, Phys. Rev. 124, 41-53 (1961).
[12] S. Maekawa et al., Physics of Transition Metal Oxides ,Series in Solid State Sciences Vol. 144 (Springer, New York, 2004), p.16.

[13] P. Giannozzi et al., http://www.quantum-espresso.org

[14] V. I. Anisimov et al., Phys. Rev. B 48, 16929 (1993).

[15] G. K. H. Madsen et al., Europhys. Lett. 69, 777 (2005).

[16] C. J. Ballhausen, Introduction to ligand field theory (McGraw-Hill, New York, 1962) p.96.

[17] A. T. Costa et al., Phys. Rev. B 78, 054439 (2008).

[18] S. Shadov et al., Europhys. Lett. 82, 37001 (2008). 